\title{
Cytomegalovirus ileitis presenting as massive rectal bleeding in infancy
}

\author{
Boniface Kawimbe, Chris Bem, Prahlad Shripad Patil, Hoshang Bharucha
}

\begin{abstract}
Two Zambian infants presented with massive rectal bleeding caused by cytomegalovirus ileitis. The first was seropositive for HIV and the second seronegative, though her mother was seropositive. Both infants died.
\end{abstract}

Clinical infection with cytomegalovirus is uncommon in the general population, though it is often found in association with HIV infection. ${ }^{1}$ We report two cases of cytomegalovirus enteritis that presented to the paediatric surgical department at the University Teaching Hospital, Lusaka, Zambia, during 1989.

\section{Case reports \\ CASE 1}

A 3 month old boy presented with a one day history of bloody diarrhoea. He had previously been in good health. On examination he was pale with a tachycardia of 152 beats/minute. The abdomen was soft and non-tender with hyperactive bowel sounds. There was a large quantity of blood clots on the nappy. Rectal examination confirmed the presence of blood. A provisional diagnosis of typhoid fever was made and in view of the life threatening haemorrhage laparotomy was done as soon as he had been resuscitated. The colon and terminal ileum were loaded with blood and the entire small bowel was covered in transmural plaque-like lesions, the heaviest concentration being in the terminal ileum. The terminal $20 \mathrm{~cm}$ of ileum were resected and intestinal continuity restored by ileocaecal anastomosis. When the resected bowel was opened the plaque-like lesions were seen to be ulcerations of varying depth. Postoperatively the infant developed a coagulopathy and wound infection, and (on the tenth day) peritonitis. At the second laparotomy multiple perforations in the remaining part of the small intestine were found and he died a few hours

Department of Surgery, University Teaching Hospital

PO Box 50110,

Lusaka,

Zambia

Boniface Kawimbe

Chris Bem

Prahlad Shripad Patil

The Queen's University, Belfast,

Northern Ireland

Hoshang Bharucha

Correspondence to:

Mr Bem.

Accepted 28 February 1991

(Arch Dis Child 1991;66:883-884) cozyme recombinant).

\section{CASE 2}

A 6 month old previously healthy girl presented with a three day history of coughing, vomiting, and bloody diarrhoea. On examination she was pale and had a tachycardia of 124 beats/minute. Her chest was normal. The abdomen was soft and non-tender with normal bowel sounds. Blood was found on rectal examination, and a provisional diagnosis of intussusception made. At laparotomy the terminal ileum and colon were filled with blood and there were plaquelike lesions similar to those in case 1 , but not as florid. A $30 \mathrm{~cm}$ length of terminal ileum was resected, with ileoileal anastomosis. The infant died two hours after laparotomy of hypovolaemic shock. She was seronegative for HIV, though her mother was seropositive (Wellcozyme recombinant).

In both cases permission for necropsy was refused. The surgical specimens showed round, oval transverse ulcers 0.5 to $1 \mathrm{~cm}$ in diameter and at different stages of evolution. Histopathological features included focal transmural inflammation rich in mononuclear cells. Fibroblasts and endothelial cells contained eosinophilic inclusions characteristic of cytomegalovirus infection. Gram, Ziehl-Neelsen, periodic acid Schiff, and Grocott-Gomori stains showed no abnormalities. Immunoperoxidase staining by the avidin biotin complex method (DAKO) confirmed the presence of cytomegalovirus. The primary antiserum used was a monoclonal anticytomegalovirus (DAKO-M757) at a dilution of 1:50 pretreated with trypsin.

\section{Discussion}

Cytomegalovirus is normally a common subclinical infection acquired at any time from intrauterine to late adult life. Clinical infection has become important as a sign of compromise of the immune system by HIV infection. ${ }^{1}$

The diagnosis of clinical infection can confidently be made if the characteristic inclusion bodies are found in biopsy material; immunohistological staining increases the sensitivity of histological examination. ${ }^{2}$ The presence of cytomegalic inclusion bodies at the sites of ulceration and inflammation in the cases reported suggests strongly that cytomegalovirus was the pathogen directly responsible for causing the devastating clinical ileitis, though it has recently been postulated that in the presence of HIV infection cytomegalovirus may have only a secondary pathogenic role. ${ }^{3}$

There is no conclusive proof that the two patients were infected with HIV. Testing for HIV infection in infants by antibody detection lacks the specificity and sensitivity that it has in adults because of the presence of maternal antibodies. ${ }^{4}$ Technically advanced methods for detecting the HIV antigen in the infants were not available, but such a remarkable presentation of an infection associated with HIV, the background prevalence of HIV infection in mothers delivering at the University Teaching Hospital, Lusaka, ${ }^{5}$ and the positive Wellcozyme test in one infant and in the mother of the other, 
suggests that both children were immunocompromised despite their previously apparent good health.

Occasional reports of cytomegalovirus infection in immunocompromised children have been reported from elsewhere, including, one other case of life threatening gastrointestinal haemorrhage. ${ }^{6}$ The cases reported here emphasise that particularly where there is the likelihood of HIV infection, cytomegalovirus enteritis must be considered in an infant presenting with rectal bleeding. It is unlikely that a definitive preoperative diagnosis will often be made, though a less acute presentation may permit time for mucosal biopsy. ${ }^{2}$ Retinoscopy may help if coexisting cytomegalovirus chorioretinitis is present. At laparotomy the presence of widespread ulcerative lesions should alert the surgeon to the possibility of the diagnosis.

In both cases the clinical problem was one of exsanguinating haemorrhage, and at laparotomy the absence of a focal lesion that could be excised. In the first case the haemorrhage continued, and though the infant survived the initial operation he died of perforation of the remaining lesions, which indicated the progressive nature of the disease. If arteriography is available it could be used to localise the site of bleeding, thereby permitting the correct operation to be done and antiviral treatment to be given to control the infection.

1 Jacobson MA, Mills J. Serious cytomegalovirus disease in the acquired immunodeficiency syndrome (AIDS). Ann Intern Med 1988;108:585-94.

2 Culpepper-Morgan JA, Kotler DP, Scholes JV, Tierney AR. Evaluation of diagnostic criteria for mucosal cytomegalic inclusion disease in the acquired immune deficiency syndrome. Am F Gastroenterol 1987;82:1264-70.

3 Morris DJ. Is human immunodeficiency virus (HIV) rather than cytomegalovirus the cause of retinitis and colitis in HIV-infected patients? Rev Infect Dis 1990;12:557-9.

4 Mok J. HIV infection in children. Br $\mathcal{J}$ Hosp Med 1990;43: 247-51.

5 Hira SK, Kamange J, Bhat GJ, et al. Perinatal transmission of HIV-1 in Zambia. BMF 1989;299:1250-2.

6 Schwartz DL, So HB, Bungarz WR, et al. A case of lifethreatening gastrointestinal haemorrhage in an infant with threatening gastrointestinal haemorrhage
AIDS. F Pediatr Surg 1989;24:313-5.
The Royal Liverpool Children's Hospital N P Madden R E Cudmore

Correspondence to: Mr N P Madden, Clarendon Wing, The General Infirmary, Leeds LS2 9NS.

Accepted 28 February 1991

(Arch Dis Child 1991;66:884-885)

\section{Scalp tumours mimicking encephaloceles}

\author{
N P Madden, R E Cudmore
}

tation had been reported as 'normal', but the serum $\alpha$ fetoprotein concentration was 2.5 times the reference value, though a week later it was found to be within the reference range.

The family history included one cousin in whom a brain tumour had been diagnosed at 5 months of age, and another who had had hydrocephalus.

Congenital tumours of the scalp are rare, three being reported in an extensive survey of congenital mesenchymal tumours. ${ }^{1}$ Two neonates with such tumours, both of which mimicked encephaloceles, have been referred to our unit during the last 20 years.

\section{Case reports}

CASE 1

A 1 day old boy was referred in 1971 with a firm lesion, $6 \mathrm{~cm}$ in diameter, and covered with skin, which lay over the left side of the lambdoid suture. It did not transilluminate, and we could not assess from skull radiographs whether there was any intracranial extension (fig 1).

At operation on the third day of life the tumour was found to be entirely extracranial, and was easily excised. Macroscopically the cut surface was uniformly grey-white. Microscopy showed a hamartomatous mass of predominantly smooth muscle. The patient was followed up for five years during which time there was no recurrence of the tumour.

CASE 2

In 1985 a 2 day old boy was referred; routine antenatal ultrasound screening at 16 weeks' ges-

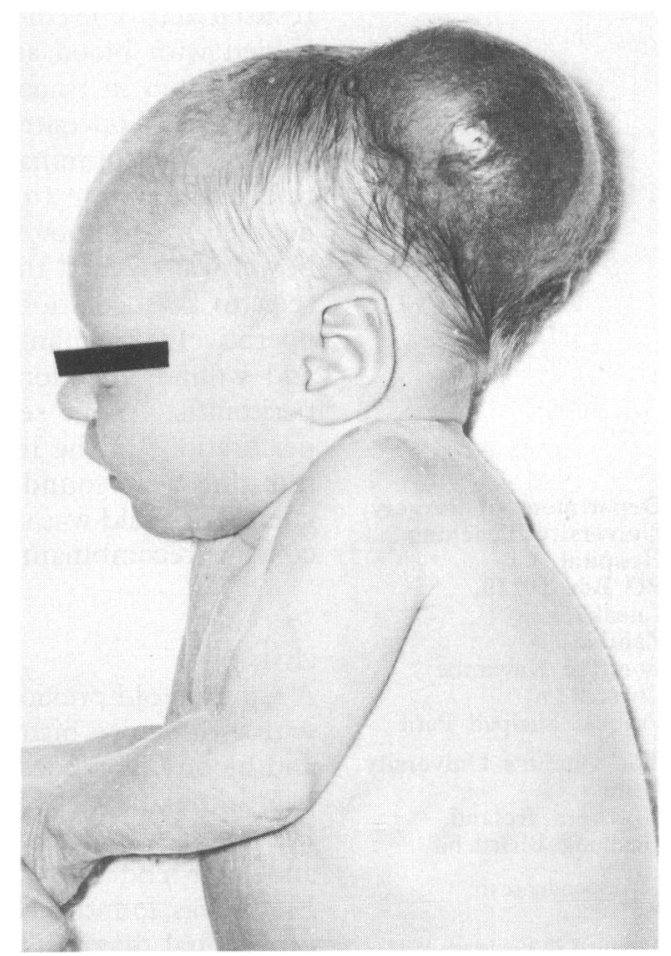

Figure 1 Case 1, aged 1 day. 